\title{
Quantile-locating functions and the distance between the mean and quantiles
}

\author{
D. Gilat* \\ School of Mathematical Sciences, Tel Aviv University \\ Ramat Aviv 69978, Israel \\ T. P. Hill** \\ School of Mathematics, Georgia Institute of Technology \\ Atlanta, GA 30332, USA
}

\begin{abstract}
Given a random variable $X$ with finite mean, for each $0<p<1$, a new sharp bound is found on the distance between a $\rho$-quantile of $X$ and its mean in terms of the central absolute first moment of $X$. The new bounds strengthen the fact that the mean of $X$ is within one standard deviation of any of its medians, as well as a recent quantile-generalization of this fact by O'Cinneide.
\end{abstract}

Key words \& Phrases: mean, median, quantiles, absolute central first moment, convex function.

\section{Introduction}

Let $X$ be a real-valued random variable with finite mean $E(X)=\mu$ and standard deviation $\sigma$. O'CINNEIDE (1990) gives an interesting proof of the fact, which he attributes to Hotelling and Solomons (1932), that the mean of $X$ is within one standard deviation of any of its medians. As observed by MaLlows and Richter (1969), even a bit more is true: the distance between the mean and any median of $X$ is bounded not only by its standard deviation (which may be infinite), but even by its (generally) smaller central first moment. Putting $m$ for any median of $X$, one obtains

$$
|E X-m| \leq E|X-m| \leq E|X-E X| \leq \sigma
$$

where the crucial second inequality in (1) is valid because, as is well known (e.g. see Bickel and Doxsum, 1977, p. 54), m minimizes the mapping $x \rightarrow E|X-x|$. Note that equalities throughout (1) are attained if $X$ is symmetric about $\mu$, two-valued and one of its values is taken for $m$.

In this note we use, for each $0<p<1$, a functional $U_{p}$ which is uniquely minimized by any $p$-quantile of $X$ to obtain a central first moment bound, which generalizes (1), on the distance between the mean of $X$ and any of its $p$-quantiles.

* Partly supported by U.S.-Israel Binational Science Foundation Grant No. 88-00005.

** Partly supported by U.S.-Israel Binational Science Foundation Grant No. 88-00005.

Partly supported by National Science Foundation Grant DMS-89-01267. 
Throughout this note, if $a$ and $b$ are real numbers, $a \vee b(a \wedge b)$ stand for their maximum (minimum) and, as is customary, $a^{+}=a \vee 0$ and $a^{-}=(-a)^{+}$. Recall that the real number $m=m_{p}$ is a $p$-quantile of $X(0<p<1)$, if $P\{X \leq m\} \geq p$ and $P\{X \geq m\} \geq 1-p$.

\section{A function uniquely minimized by $p$-quantiles}

Given a random variable $X$ with finite mean, for $0<p<1$, let the functions $U_{p, X}=U_{p}$ : $\mathbb{R} \rightarrow \mathbb{R}$ be defined by

$$
U_{\rho}(x)=p E(X-x)^{+}+(1-p) E(X-x)^{-} .
$$

Note that $U_{1 / 2}(x)=(1 / 2) E|X-x|$; the next proposition records two useful properties of $U_{p}$.

Proposition 1. For each $0<p<1, U_{p}$ is convex, and $U_{p}$ uniquely determines the distribution of $X$.

Proof: The convexity of $U_{p}$ follows easily from the convexity (for each $y$ ) of the maps $x \rightarrow(y-x)^{+}$and $x \rightarrow(y-x)^{-}$. The usual technique of integration by parts yields

$$
U_{p}(x)=p \int_{x}^{\infty} P\{X>t\} \mathrm{d} t+(1-p) \int_{-\infty}^{x} P\{X \leq t\} \mathrm{d} t .
$$

Thus $U_{\rho}$ is differentiable at continuity points of $P\{X \leq x\}$ and if $x$ is such a point, then the derivative $U_{p}^{\prime}$ satisfies

$$
U_{\rho}^{\prime}(x)=-p P\{X>x\}+(1-p) P\{X \leq x)=P(X \leq x)-p=(1-p)-P(X>x) .
$$

Since the set of such $x$ 's is dense in $\mathbb{R}$, the distribution of $X$ can be recovered from (4).

The property of $U_{p}$ which will be used in deriving the mean-quantile bound mentioned in the introduction is that $U_{p}$ is a $p$-quantile-locator in the sense of the following proposition (cf. also FERGUSON, 1967, Exercise 3, page 51, where uniqueness is not claimed). Note that this includes the well-known inequality (used in (1)) that the median minimizes $E|X-x|=2 U_{1 / 2}(x)$.

Proposition 2. For every integrable random variable $X$ and every $p \in(0,1)$, the function $U_{p}$ is uniquely minimized by any $p$-quantile of $X$. That is, $U_{p}(x)=\min \left\{U_{p}(y): y \in \mathbb{R}\right\}$ if and only if $x$ is a p-quantile of $X$.

Proof: Since $U_{\rho}$ is nonnegative, convex and unbounded on both the positive and negative rays of $\mathbb{R}$, it has a minimum. If, in addition, $U_{p}$ is differentiable (which is the case when the distribution of $X$ is continuous) then setting the derivative $U_{\rho}^{\prime}(x)=0$ in formula (4) completes the proof that $U_{p}$ is minimized by every p-quantile. In general, when the distribution of $X$ may have atoms, proceed as follows. 
First apply integration by parts to rewrite $U_{p}$, defined in (2), in the forms

$$
\begin{aligned}
U_{p}(x) & =p(E X-x)+E(X-x)^{-} \\
& =p(E X-x)+\int_{-\infty}^{x} P\{X \leq t\} \mathrm{d} t \\
& =-(1-p)(E X-x)+E(X-x)^{+} \\
& =-(1-p)(E X-x)+\int_{x}^{\infty} P\{X>t\} \mathrm{d} t .
\end{aligned}
$$

Next, distinguish between two cases. If $x \geq m_{p}$ use (5i) to obtain

$$
\begin{aligned}
U_{p}(x) & =p(E X-x)+\int_{-\infty}^{m_{p}} P\{X \leq t\} \mathrm{d} t+\int_{m_{p}}^{x} P\{X \leq t\} \mathrm{d} t \\
& \geq p(E X-x)+\int_{-\infty}^{m_{p}} P\{X \leq t\} \mathrm{d} t+p\left(x-m_{p}\right) \\
& =p\left(E X-m_{p}\right)+E\left(X-m_{p}\right)^{-}=U_{p}\left(m_{p}\right),
\end{aligned}
$$

where the inequalty is valid because $m_{p}$ is a $p$-quantile of $X$, and the last equality follows from (5i). The proof for the case $x<m_{p}$ is similar using (5ii). This completes the proof that $U_{p}$ is minimized by any $p$-quantile.

To see the converse note that if $x$ is strictly larger than the largest $p$-quantile, then the inequality in (6) is strict. Similarly, using (5ii), a strict inequality is obtained when $x$ is strictly smaller than the smallest $p$-quantile. Thus the minimum of $U_{\beta}$ is attained only at $p$-quantiles.

\section{Distance between the mean and quantiles}

For an integrable random variable $X$ and each $0<p<1$, let $V_{p, X}=V_{p}: \mathbb{R} \rightarrow \mathbb{R}$ be defined by

$$
V_{\rho}(x)=p(E X-x)^{+}+(1-p)(E X-x)^{-},
$$

and let

$$
\Delta_{p}=U_{p}-V_{p}
$$

where $U_{p}$ is as in (2).

Note that $V_{p}$ is piecewise linear with slope $p$ to the left of $E X$ and slope $1-p$ to its right.

Some useful properties of $\Delta_{p}$ are recorded in the following lemma.

Lemma. For each $0<p<1$,
(i) $\Delta_{p}(x)=\left\{\begin{array}{ll}E(X-x)^{-}, & x \leq E X \\ E(X-x)^{+}, & x \geq E X\end{array}\right.$ independently of $p$
(ii) $0 \leq \Delta_{\rho}(x) \rightarrow 0$ as $|x| \rightarrow \infty$; and
(iii) $\int_{-\infty}^{\infty} \Delta_{p}(x) \mathrm{d} x=\frac{1}{2} \operatorname{Var} X$ (whether finite or not). 
Proof: (i) By definition,

$$
\begin{aligned}
\Delta_{p}(x)= & U_{p}(p)-V_{p}(x)=p\left\{E(X-x)^{+}-(E X-x)^{+}\right\} \\
& \left.+(1-p)\left\{E(X-x)^{-}-E X-x\right)^{-}\right\} \\
= & p\left\{\left[E(X-x)^{+}-E(X-x)^{-}\right]-\left[(E X-x)^{+}-(E X-x)^{-}\right]\right\} \\
& +\left\{E(X-x)^{-}-(E X-x)^{-}\right\} \\
= & p\{(E X-x)-(E X-x)\}+\left\{E(X-x)^{-}-(E X-x)^{-}\right\} \\
= & \left\{E(X-x)^{-}-(E X-x)^{-}\right\} \\
= & \begin{cases}E(X-x)^{-}, & x \leq E X \\
E(X-x)^{+}, & x \geq E X .\end{cases}
\end{aligned}
$$

(ii) The inequality follows from (i) (or from Jensen). The asymptotic statement follows from (i) using monotone convergence.

(iii) Assume, without loss of generality, that $E X=0$. By (i)

$$
\int_{-\infty}^{\infty} \Delta_{p}(x) \mathrm{d} x=\int_{-\infty}^{0} E(X-x)^{-} \mathrm{d} x+\int_{0}^{\infty} E(X-x)^{+} \mathrm{d} x .
$$

Applying integration by parts twice and using Fubini in between to change the order of integration, one obtains

$$
\begin{aligned}
\int_{-\infty}^{\infty} \Delta_{p}(x) \mathrm{d} x & =\int_{-\infty}^{0} t P\{X<t\} \mathrm{d} t+\int_{0}^{\infty} t P\{X>t\} \mathrm{d} t \\
& =(1 / 2)\left\{E\left(X^{-}\right)^{2}+E\left(X^{+}\right)^{2}\right\}=\left(\frac{1}{2}\right) E X^{2} .
\end{aligned}
$$

REMARK. Applying (iii) with $p=\frac{1}{2}$ it follows that

$$
\int_{-\infty}^{\infty}\{E|X-x|-|E X-x|\} \mathrm{d} x=\operatorname{Var} X
$$

Finally, Proposition 2 and the above properties of $\Delta_{p}$ will be used to obtain bounds on the distance between any $p$-quantile of a random variable and its mean in terms of its central absolute first moment. These bounds are analogous to the standard deviation bounds of DHARMADHIKARI (1991)

$$
E X-\sigma \sqrt{q / p} \leq m_{p} \leq E X+\sigma \sqrt{p / q}
$$

which both generalize (1) and strengthen the symmetric version of O'CINNEIDE.(1990)

$$
\left|E X-m_{p}\right| \leq \sigma \sqrt{\max \{p / q, q / p\}}
$$


THEOREM 1. For $0<p<1$, let $m_{p}$ be a $p$-quantile of the random variable $X$. If EX is finite, then (letting $q=1-p$ )

$$
E X-(1 / 2 p) E|X-E X| \leq m_{p} \leq E X+(1 / 2 q) E|X-E X|,
$$

and these bounds are attained.

Proof: If $m_{p} \leq E X$ then

$$
\begin{aligned}
p\left(E X-m_{p}\right) & =V_{p}\left(m_{\rho}\right) \leq U_{p}\left(m_{p}\right) \leq U_{p}(E X) \\
& =p E(X-E X)^{+}+(1-p) E(X-E X)^{-}=(1 / 2) E|X-E X|,
\end{aligned}
$$

where the first equality follows from (7), the first inequality from Lemma (ii), the second finequality from Proposition 1 , the second equality from the definition of $U_{p}$, and the last equality since $E(X-E X)^{+}=E(X-E X)^{-}=(1 / 2) E|X-E X|$. If $m_{p} \geq E X$, use the same argument with the fact from (7) that $(1-p)\left(m_{p}-E X\right)=V\left(m_{p}\right)$. Equality is easily seen to be attained if $X$ takes only two values $a<b$ and $P(X=a)=p$.

\section{Acknowledgement}

The authors are grateful to the Mathematics Department of the Free University of Amsterdam and especially to Professor Piet Holewijn, for their hospitality during the spring of 1991 where this research was begun, and to Professor Ester Samuel-Cahn for pointing out the Ferguson reference.

\section{References}

BiCKel, P. J. and K. A. Doxsum (1977), Mathematical statistics, Holden-Day, San Francisco.

Dharmadhikari, S. (1991), Bounds on quantiles: a comment on O'Cinneide, The American Statistician 45, 257-58.

Ferguson, T. S. (1967), Mathematical statistics, Academic Press, New York.

Hotelling, H. and L. M. Solomons (1932), The limits of a measure of skewness, The Annuals of Mathematical Statistics 3. 141-142.

Mallows, C. and D. Richter (1969), Inequalities of Chebyschev type involving conditional expectations, The Annuals of Mathematical Statistics 40,1922-1932.

O'CINNEIDE, C. A. (1990), The mean is within one standard deviation of any median, The American Statistician 44, 292-293. 